\title{
Integrating Electrical Substations within the IoT using IEC 61850, CoAP and CBOR
}

\author{
Markel Iglesias-Urkia, Diego Casado-Mansilla, Simon Mayer, Josu Bilbao, and Aitor Urbieta
}

\begin{abstract}
Electrical substations are crucial elements of Smart Grids where they are mainly responsible for voltage transformations. However, due to the integration of distributed energy resources in the grid, substations now have to provide additional grid management capabilities which in turn require supervision and automation solutions for large low-voltage grids. A recurring challenge in such deployments are siloed systems that are due to non-interoperable communication protocols across substations: although most substations' communication is based on the International Electrotechnical Commission (IEC) 61850 standard, deployed legacy protocols lag behind modern communication technologies in terms of performance, hindering the full transition to lightweight protocols.

This paper demonstrates that IEC 61850 can be fully mapped to the Constrained Application Protocol (CoAP) in combination with the Concise Binary Object Representation (CBOR) format while improving system performance compared to existing alternatives (e.g. WS-SOAP and HTTP). On average, CoAP+CBOR needs $44 \%$ and $18 \%$ of the message size and $71 \%$ and $85 \%$ of the time compared to systems based on HTTP and WS* Web Services, respectively - this is especially relevant for resource-constrained devices and networks in electrical grids. In addition, CoAP is based on the Representational State Transfer (REST) architectural style, which supports system integration and interoperability through uniform identification and interaction. This approach fosters the standard-compliant integration of legacy platforms with modern substations as well as current IoT systems in neighboring domains such as building management and infrastructure automation systems.
\end{abstract}

Index Terms-Benchmarking, CBOR, CoAP, HTTP, IEC 61850, Internet of Things, IoT, Smart Grids, WS-SOAP.

\section{INTRODUCTION}

O VER the past few years, more active components (e.g., photovoltaics (PV), residential batteries, and home automation systems) are being added to electric power distribu-

Manuscript received September 1, 2018; revised February 25, 2019. This work has received funding from the Electronic Component Systems for European Leadership Joint Undertaking under the MegaM@Rt2 project (Grant agreement No. 737494). This Joint Undertaking receives support from the European Union's Horizon 2020 research and innovation program and from Sweden, France, Spain, Italy, Finland \& Czech Republic. Besides, this work has been partially supported by the Basque Government through the Elkartek program under the TEKINTZE project (Grant agreement No. KK2018/00104).

Markel Iglesias-Urkia, Dr. Josu Bilbao and Dr. Aitor Urbieta are with IK4-Ikerlan, Arrasate-Mondragón, 20500, Spain. (e-mails: \{miglesias,jbilbao,aurbieta\}@ikerlan.es).

Dr. Diego Casado is with DeustoTech - Societal Challenges, Bilbao, 48007, Spain. (e-mail: dcasado@deusto.es).

Prof. Dr. Simon Mayer is with the University of St. Gallen and with ETH Zurich, Switzerland. (e-mail: simon.mayer@unisg.ch).

Copyright (c) 2019 IEEE. Personal use of this material is permitted. However, permission to use this material for any other purposes must be obtained from the IEEE by sending a request to pubs-permissions@ieee.org. tion grids. Some of these components actively generate energy, which leads to a paradigm shift in power grids: from the pure distribution of energy among consumers to the need for active management of the resulting "Smart Grids (SGs)". Traditional centralized distribution does not require extensive control hence, distribution grids used to be operated passively; however, with the inclusion of such Distributed Energy Resources (DERs), SG control is becoming increasingly complex [1] consequently, SG operators are more and more pushing for the integration of Supervisory Control and Data Acquisition (SCADA) functionality to enable advanced management of the power grid.

To enable SCADA functionality in the power grid, operators first need to equip distribution substations with the relevant sensors, and connect them to their back-end infrastructure. In order to guarantee the successful retrofitting of the legacy scenarios, the following features must be addressed: i) low deployment cost, ii) minimal maintenance effort, iii) low latency, and iv) good performance under low bandwidth networks. The solutions must also take account of the limited amount of space within substations - as a consequence, industry is turning toward wireless communication technologies, especially for remote deployments: Wireless Personal-Area Networks (WPANs) are suggested to connect devices within substations; and Low-Power Wide-Area Networks (LPWANs) are expected to link substations to back-end systems ${ }^{1}$, in particular for remote installations [2]. The retrofitting of SGs with Internet of Things (IoT) technology depends on solutions that are, at the same time, standard (i.e., compliant with IEC 61850), interoperable on the application layer (e.g., compatible with REpresentational State Transfer (REST)) and able to operate within constrained settings that feature only limited Quality of Service (QoS). Based on these requirements, after surveying different mappings of the IEC 61850 to different communication protocols, we proposed [3] and evaluated [4] a mapping of the IEC 61850 standard to the Constrained Application Protocol (CoAP).

In this paper, we implement and extend the work carried out so far, changing the resource representation format to the Concise Binary Object Representation (CBOR), completing the full IoT-stack integration with $\mathrm{CoAP}+\mathrm{CBOR}$ and also adding an extension to CoAP's subscription mechanism presented in [5]. This extension for the subscription mechanism overcomes the issues assessed in [4], where more advanced features for subscribing were considered necessary. This results in a

\footnotetext{
${ }^{1}$ Interestingly, power line communication technologies are not suitable in this case, as indeterminate impedance variations and cross-talk make them too unreliable [2].
} 
communication architecture for SGs components that can cope with low QoS while remaining interoperable with back ends, thereby supporting SCADA-like functionality in distribution grids. In this way, the presented system does not only feature a communication mechanism that has been conceived for resource-constrained devices, but also the preferred encoding for constrained environments, thereby making a compelling case for real-world adoption.

The rest of the article is organized as follows: the following section discusses the related work, especially on IEC 61850 mappings and our enhancements to the CoAP Observe mechanism. Subsequently, we have included a brief primer on the technical background of the different technologies that are relevant in the context of our work. Next, we discuss the design of our system in Section IV and its implementation in Section V. After the implementation, we present the results from our evaluation in Section VI. Finally, we discuss our results and potential future research directions in Section VII.

\section{RELATED WORK}

In the following, we describe the state of the art of the currently proposed IEC 61850 mappings and CoAP's Observe extensions. There are several studies in the scientific literature that provide a high level analysis of the features using different approaches in SG environments [6] [7]. However, to the best of our knowledge, there is a lack of studies that focus on analyzing middlewares based on IEC 61850.

\section{A. IEC 61850 mappings}

In [3] and [4], a review of the existing mappings of the IEC 61850 standard to different application-layer protocols is described. The IEC 61850 standard proposes to use the Manufacturing Message Specification (MMS) as applicationlayer protocol, but it also encourages to use other protocols providing the freedom to developers to choose the most suitable for each scenario. The same applies to other standards such as IEC 61400 that proposes Web-Services (WS) - Simple Object Access Protocol (SOAP) for its mapping [8]; in addition to the IEC recommendations, several other mappings have been proposed: Common Object Request Broker Architecture (CORBA) [9], combining CORBA and Data Distribution Service (DDS) [10], [11] and applying DDS [12]. Using HyperText Transfer Protocol (HTTP) was first proposed in [13] and later expanded in [14]. A publish/subscribe communication approach was proposed in [15]. While these proposals feature widely used technologies (HTTP and XMPP), they incur a high communication overhead due to the text-based nature of HTTP and the complexity and overhead of exchanging presence information within eXtensible Messaging and Presence Protocol (XMPP), as well as it being based on the rather verbose eXtensible Markup Langage (XML). Shin et al. propose in [16] the first approach to use a protocol specifically created for IoT systems, CoAP. However, the authors map just a reduced set of functions and they do not follow a RESTful approach, as they use different resources for PUT and GET requests.
The network constraints presented in the introduction make it necessary to find ways to minimize the network requirements, and for that, in [3], the authors propose a more extensive approach following the REST architectural style to later implement and validate it in [4]. However, the proposed mapping uses CoAP in the current state of development and presents certain limitations in terms of subscription mechanisms for publish/subscribe communications that are tackled in the present work. The two main limitations are i) when updating a resource, a client cannot subscribe to that resource with the same request, and ii) a client might not need the resource representation when requesting it, just when an event that triggers a notification happens.

\section{B. CoAP Observe enhancements}

From the issues raised in [3] and [4], new subscription mechanisms for CoAP's Observe extension were proposed in [5]. Although some work was previously done to enhance the publish/subscribe paradigm of the CoAP standard, all the previous work was about optimizing notifications rather than streamlining the subscription mechanism itself - we discuss this point in greater depth in the following paragraph.

The Internet Engineering Task Force's (IETF) Constrained RESTful Environments (CoRE) group [17] proposes different documents for communications in resource-constrained devices and networks. One of them is Observing Resources in the Constrained Application Protocol (RFC 7641) for using CoAP as a publish/subscribe communication protocol. This CoAP Observe extension includes two different parts, the subscription and the notification mechanisms. [5] surveyed the proposals for improving CoAP's Observe extension and concluded that some work has been done to enhance CoAP notifications, but enhancements for the subscription mechanisms have been limited. Dynamic Resource Linking for Constrained RESTful Environments (Dynlink) [18] and Publish-Subscribe Broker for the Constrained Application Protocol (CoAP) (CoAP-PubSub) [19] propose enhancements to improve the generation and delivery of notifications. The former defines some parameters to allow a client to select which notifications it wants to receive by using a fixed set of parameters: pmin - minimum time between notifications; pmax - maximum time between notifications; $s t$ - step in the value; $g t$ - upper limit value; $l t$ lower limit value; and band - a bounded or unbounded value range. The latter proposes to mimic other publish/subscribe protocols by using a broker as an intermediary and thereby decoupling the clients and servers and allowing them to enter an idle or sleeping mode. Clients can push data through PUT or POST requests and request the data through notifications or GET requests.

Ketema et al. [20] proposed using new CoAP options to define different criteria for notification generation; Mietz et al. [21] propose a new High-Level State Option to give semantic meaning to the values, e.g., cold and warm for temperature values; Tanganelli et al. [22] limit the number of notifications using proxies to optimize notification periods, also enabling resource-constrained devices to handle many subscribers and Ludovici et al. [23] propose to specify QoS parameters with 
priorities for the notifications. In [24], [25] and [26], the authors propose a framework for planning registration states to aggregate and schedule the notification-sending process, using timeliness fairness to that schedule, and finally add a heuristic algorithm to improve the registration steps. Ishaq et al. [27] propose an entity named Entity Manager to improve the interaction of CoAP's Observe and Group extensions, aggregating the notifications while Choi et al. [28] propose to use a clustering approach, grouping the sensors in clusters and using one as the head.

All mentioned publications focus on reducing the number of notification deliveries, aggregating or skipping notifications when possible. However, to fulfill the needs presented in the mapping of the IEC 61850 to CoAP, it is required to enhance the subscription part of the Observe extension [5], using new CoAP option and response codes to fulfill the following requirements:

1) Enable subscriptions with PUT/POST.

2) Drop requirement to include resource representation on every request.

3) Enable subscriptions through proxy resources.

\section{TECHNICAL BACKGROUND}

After the critical analysis of the related work and briefly presenting new CoAP options we use in this work, in this section we present the technical background for the conducted research work, with an introduction to the IEC 61850 standard and the communication protocols we analyze, focusing on CoAP.

\section{A. The IEC 61850 Standard}

IEC 61850 is a standard of the IEC for modelling, controlling and monitoring electrical substations in power grids. It defines a model (Information Model) and the communications with mappings to specific communication protocols, along with services to interact with the model [29].

The Information Model consists of the Basic Information Model (BIM) and Control Blocks for Additional Functions (CBs). The BIM describes the organization of the elements and their information and the CBs are blocks to set and manage additional functionality. For this work, we included the reporting blocks and Fig. 1 shows the elements of the $\mathrm{BIM}$ and the CBs, with the unimplemented CBs in grey.

The BIM includes the following elements:

- Servers expose the system to outside elements.

- Logical Devices (LD) virtually represent real devices.

- Logical Nodes ( $L N)$ virtually abstract each functionality of the CBs. There is a special Logical Node Zero (LLNO) to represent the common data of the LD.

- Data represents specific information about the physical world.

- DataAttributes represent each piece of information of a Data item.

- Dataset represents a set of Data items.

The services the CBs cover are functions for Reporting, Logging, Configuration, Eventing and Sampled Value Transmission. The IEC 61850 tool used for this project (described

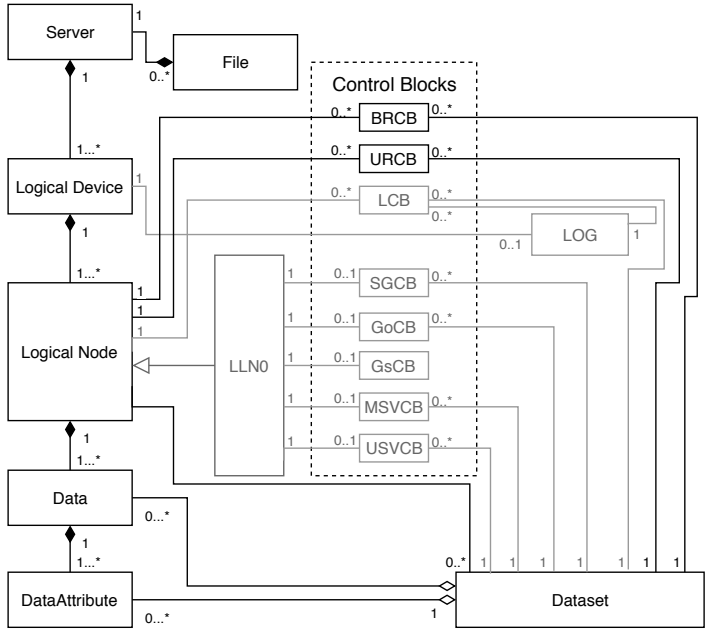

Fig. 1. Basic information model of the IEC 61850 standard.

in Section V) only implements Reporting CBs, i.e., Buffered $(B R C B)$ and Unbuffered (URCB). Due to the fact that the HTTP and WS-SOAP implementations have been generated with that same tool, the work presented in this paper is limited to those CBs. However, with the functions that are implemented in this work, all types of possible requests to interact with the model can be analyzed, i.e., retrieving, creating, updating or deleting data or getting a notification when some event occurs. All the elements, both in the BIM and in the CBs have an absolute reference to identify them through the entire system.

\section{B. Communication Protocols}

For this work, a CoAP mapping for IEC 61850 has been implemented, building on top of enhancements for CoAP's Observe subscription mechanisms presented in [5]. Next, this implementation has been compared to HTTP and WSSOAP approaches in terms of performance. In this section we summarize the main features of the three mentioned protocols, i.e., HTTP, WS-SOAP and CoAP.

1) HTTP: HTTP is the predominant application-layer communication protocol of the World Wide Web. It defines a uniform interface for Web resources that consists of several types of request methods called verbs (e.g., GET, POST, PUT, etc.). Resources themselves are identified using Uniform Resource Identifiers (URI); the interaction with resources is carried out by transmitting resource representations, where a content negotiation mechanism is used to determine the representation format (e.g., HTML, XML or JSON).

2) WS-SOAP: SOAP specifies a messaging protocol for exchanging structured information and is part of the WS-* protocol stack. In WS-SOAP, client requests and service responses are transmitted through HTTP POST requests, i.e. using HTTP as a pure transport protocol. Web Services Description Language (WSDL) is used to declare the services' functionality and interfaces for the clients to know the available services. For addressing schemes, security parameters, and discovery services other standards of the WS-* family can be used. 
Priyantha et al. [30] did some work to adapt WS-SOAP to resource-constrained devices.

3) CoAP: The IETF CoRE Working Group standardized The Constrained Application Protocol (CoAP) [31] as RFC 7252 in 2014. To extend CoAP's capabilities, other related RFCs have been or are on the way of being standardized, including i) Constrained RESTful Environments (CoRE) Link Format $^{2}$ for defining content formats; ii) Observing Resources in $\operatorname{CoAP}^{3}$ to enable publish/subscribe communications; and iii) Block-Wise Transfers in the Constrained Application Protocol $(C o A P)^{4}$ to be able to send larger payloads in chunks.

Similar to HTTP, CoAP is a communication protocol that can be used for REST interactions in client-server communication. CoAP defines a subset of HTTP request methods, i.e., GET, POST, PUT and DELETE. With these request methods, CoAP is able to perform the basic "CRUD" functions on resources: Create, Read, Update, and Delete.

- GET is used to retrieve a resource representation. GET is safe, meaning it does not modify the resource.

- DELETE removes a resource.

- POST is used to create a new resource from a transmitted resource representation or to update an existing resource.

- PUT is used to update a resource, and is idempotent. ${ }^{5}$

One major difference between HTTP and CoAP is that the latter runs on top of the User Datagram Protocol (UDP) instead of the Transmission Control Protocol (TCP). For response codes, it uses a subset of HTTP's codes, but also new ones defined specifically for CoAP. 2.XX codes are used for correct responses, 4.XX for client errors and 5.XX for server errors. When running without security, CoAP uses the 5683 port and if used on top of DTLS for security, port 5684. The resources can be addressed with URIs, which also can include queries, in the form of coap://host[:port][path][?query].

Contrary to TCP, UDP does not guarantee reliability in packet delivery and ordering, so CoAP uses a simple mechanism on the application layer, using CONfirmable (CON) and NON-Confirmable (NON) messages, the former requiring an acknowledgment and the latter using a fire and forget approach.

Another important property of CoAP is its Observe extension. This allows to use push notifications, resulting in a publish/subscribe communication pattern. To do this, a client sends an extended GET request, with the observe option activated - the server then adds the client to its list of subscribers for that resource and keeps it updated about changes of the resource. The server decides what a change in the resource is, e.g., value changes, value changes out of a predefined bound or periodical timeouts.

\section{The Concise Binary Object Representation (CBOR)}

The Concise Binary Object Representation (CBOR) [32] is the representation format that the IETF proposes for IoT

\footnotetext{
${ }^{2} \mathrm{https}: / /$ tools.ietf.org/html/rfc6690

${ }^{3} \mathrm{https}: / /$ tools.ietf.org/html/rfc7641

${ }^{4}$ https://tools.ietf.org/html/rfc7959

${ }^{5}$ An idempotent operation can be applied multiple times without modifying the resource state beyond the initial application of the operation.
}

applications. It is defined in the RFC 7049 and is a binary serialization data format that aims to be coded with a small code footprint, small message size and extensibility with no need for negotiating the version. This makes CBOR a good fit for IoT environments, completing a lightweight stack with UDP, CoAP and CBOR [33].

In [34], the authors compare several data serialization formats in SG environments, and show that CBOR representation of data needs less bytes than both JSON and XML. The IETF also uses CBOR to represent its Link Format [35], which is used in CoAP. In this work, we use CBOR to reduce the total number of bytes transmitted in our CoAP mapping for IEC 61850 .

\section{DESIGN OF THE SYSTEM}

The present section describes the designed IEC 61850 to CoAP mapping and the proposed CoAP enhancement to optimize the Observe mechanism.

\section{A. Mapping of the IEC 61850 to CoAP}

As discussed in Section II, current IEC 61850 mappings are based on legacy protocols that have two main drawbacks:

- They are protocols that are not oriented to devices with limited resources or to low bandwidth data connection, which causes communication problems due to the large overhead generated by the messages sent with respect to the real information to be sent.

- These legacy protocols present interoperability problems with existing IoT protocols, making it difficult to carry out large-scale SG deployments, especially if devices and services need to be integrated across vertical application domains.

The CoAP protocol has been used as the basis for the mapping. CoAP was selected because it does not only implement a client-server approach, but also offers a publish/subscribe mechanism. This makes CoAP clearly aligned with the services described by the standard, since several of them are client-server (basic services) and others (e.g., reporting) are aligned with a publish/subscribe approach. A comparison is provided in [36], in which the advantages offered by CoAP over another widely used IoT protocol, namely Message Queuing Telemetry Transport (MQTT), are described. This comparison attempts to analyze the different communication patterns that can be found in IoT systems and the degree of compliance offered by these protocols.

The IEC 61850 standard defines 68 services, most of which have been mapped in [3], but a subset of the most relevant ones have been implemented and evaluated for this work. The services that have been implemented include different types of messages, including getters, setters, notifications and subscriptions. Table I summarizes the implemented services, giving the names of the IEC 61850 functions together with the mapped URI and CoAP request method, along with an identifier for the result table and charts.

Contrary to CoAP, HTTP does not have an extension to enable publish/subscribe communications. This is why the HTTP and WS-SOAP implementations have a particularity on 
TABLE I

THE SET OF IEC 61850 SERVICES MAPPED TO THE COAP COMMUNICATION PROTOCOL.

\begin{tabular}{|c|c|c|c|c|}
\hline Function & URI & Method & Id & Notes \\
\hline \multicolumn{5}{|c|}{ Basic } \\
\hline \multicolumn{5}{|c|}{ Server } \\
\hline GetServerDirectory & coap://\{host $\} / L D s$ & GET & 1 & Logical Devices \\
\hline GetServerDirectory & coap://\{host $\} /$ Files & GET & 2 & Files \\
\hline \multicolumn{5}{|c|}{ Logical Device } \\
\hline GetLogicalDeviceDirectory & coap://\{host $\} / L D s /\{L D\}$ & GET & 3 & \\
\hline \multicolumn{5}{|c|}{ Logical Node } \\
\hline GetLogicalNodeDirectory & coap: $/ /\{$ host $\} / \mathrm{LDs} /\{\mathrm{LDs}\} /\{\mathrm{LN}\} /\{$ ACSIClass $\}$ & GET & $\begin{array}{l}4 \\
5 \\
6 \\
7\end{array}$ & $\begin{array}{l}\text { Datas } \\
\text { Datasets } \\
\text { BRCBs } \\
\text { URCBs }\end{array}$ \\
\hline GetAllDataValues & coap://\{host $\} / \mathrm{LDs} /\{\mathrm{LDs}\} /\{\mathrm{LN}\} /$ AllValues $[? \mathrm{FC}=\{\mathrm{fc}\}]$ & GET & $\begin{array}{l}8 \\
9 \\
10\end{array}$ & $\begin{array}{l}\text { Big payload } \\
\text { Medium payload } \\
\text { Small payload }\end{array}$ \\
\hline \multicolumn{5}{|c|}{ Data } \\
\hline GetDataValues & coap: $/ /\{$ host $\} /$ LDs $/\{$ LDs $\} /\{$ LN $\} /$ Datas $/\{$ Data $\}$ & GET & 11 & \\
\hline SetDataValues & coap $: / /\{$ host $\} /$ LDs $/\{$ LDs $\} /\{$ LN $\} /$ Datas $/\{$ Data $\}$ & PUT & 12 & \\
\hline GetDataDirectory & coap: $/ /\{$ host $\} /$ LDs $/\{$ LDs $\} /\{$ LN $\} /$ Datas/\{Data $\} /$ Directory & GET & 13 & \\
\hline GetDataDefinition & coap: $/ /\{$ host $\} /$ LDs $/\{$ LDs $\} /\{$ LN $\} /$ Datas $/\{$ Data $\} /$ Definition & GET & 14 & \\
\hline \multicolumn{5}{|c|}{ Dataset } \\
\hline GetDataSetValues & coap: $/ /\{$ host $\} /$ LDs $/\{$ LDs $\} /\{$ LN $\} /$ Datasets $/\{$ Dataset $\}$ & GET & 15 & \\
\hline GetDataSetDirectory & coap $: / /\{$ host $\} /$ LDs $/\{$ LDs $\} /\{$ LN $\} /$ Datasets $/\{$ Dataset $\} /$ Directory & GET & 16 & \\
\hline \multicolumn{5}{|c|}{ Reporting } \\
\hline \multicolumn{5}{|c|}{$\mathrm{BRCB}$} \\
\hline Report & coap://\{host $\} / \mathrm{LDs} /\{\mathrm{LDs}\} /\{\mathrm{LN}\} /\{$ BRCBs $\} /$ BRCB/Reports & Notification & $\begin{array}{l}17 \\
18\end{array}$ & $\begin{array}{l}\text { Big payload } \\
\text { Small payload }\end{array}$ \\
\hline GetBRCBValues & coap://\{host $\} /$ LDs $/\{$ LDs $\} /\{$ LN $\} /\{$ BRCBs $\} /$ BRCB & GET & 19 & \\
\hline SetBRCBValues & coap://\{host $\} / \mathrm{LDs} /\{\mathrm{LDs}\} /\{\mathrm{LN}\} /\{$ BRCBs $\} / \mathrm{BRCB}$ & PUT & 20 & \\
\hline if enable & coap: $/ /\{$ host $\} /$ LDs/ $/$ LDs $\} /\{$ LN $\} /\{$ BRCBs $\} /$ BRCB/Reports & GET+Obs & 21 & \\
\hline \multicolumn{5}{|c|}{ URCB } \\
\hline Report & coap://\{host $\} / \mathrm{LDs} /\{\mathrm{LDs}\} /\{\mathrm{LN}\} /\{\mathrm{URCBs}\} / \mathrm{URCB} /$ Report & Notification & $\begin{array}{l}22 \\
23\end{array}$ & $\begin{array}{l}\text { Big payload } \\
\text { Small payload }\end{array}$ \\
\hline GetURCBValues & coap: $/ /\{$ host $\} / \mathrm{LDs} /\{\mathrm{LDs}\} /\{\mathrm{LN}\} /\{\mathrm{URCB}\} / \mathrm{URCB}$ & GET & 24 & \\
\hline SetURCBValues & coap://\{host $\} / \mathrm{LDs} /\{\mathrm{LDs}\} /\{\mathrm{LN}\} /\{\mathrm{URCBs}\} / \mathrm{URCB}$ & PUT & 25 & \\
\hline if enable & coap://\{host $\} / \mathrm{LDs} /\{\mathrm{LDs}\} /\{\mathrm{LN}\} /\{$ URCBs $\} / \mathrm{URCB} /$ Report & GET+Obs & 26 & \\
\hline \multicolumn{5}{|c|}{$\begin{array}{l}\text { Additional Services } \\
\end{array}$} \\
\hline \multicolumn{5}{|c|}{ Access Control } \\
\hline Associate & coap://\{host $\} /$ Associations & POST & 27 & \\
\hline Abort & coap://\{host $\} /$ Associations $/\{$ AssID $\}$ ?action=abort & DELETE & 28 & \\
\hline Release & coap: $/ /\{$ host $\} /$ Associations $/\{$ AssID $\}$ ?action=release & DELETE & 29 & \\
\hline \multicolumn{5}{|c|}{ File Transfer } \\
\hline GetFile & coap://\{host $\} /$ Files $/\{$ File $\}$ & GET & $\begin{array}{l}30 \\
31\end{array}$ & $\begin{array}{l}\text { Big file } \\
\text { Small file }\end{array}$ \\
\hline SetFile (update existing) & coap://\{host $\} /$ Files/\{File\} & PUT & 32 & \\
\hline SetFile (new file) & coap://\{host $\} /$ Files & POST & 33 & \\
\hline DeleteFile & coap://\{host $\} /$ Files/\{File $\}$ & DELETE & 34 & \\
\hline GetFileAttributeValules & coap://\{host $\} /$ Files/\{File\}/Status & GET & 35 & \\
\hline
\end{tabular}

how the reporting services work. In order to get notifications, separate clients and servers had to be created. The servers store generated reports and enable clients to obtain reports through polling. In CoAP, this is not necessary, since the Observe extension allows to use publish/subscribe communication and easily send the reports when they are generated.

To be able to integrate the mapping into the IEC 61850 tool, two changes have been considered necessary compared to the mapping presented in [3]: First, SetFile has been divided into two different functions depending on whether the file previously existed or not. If it did, a PUT request is sent to the resource pointing to the file, and if it did not, a POST request is sent to /Files. Second, in GetDataValues, a query with the functional constraint is necessary in order to integrate the solution with the existing system.
Regarding other improvements of the mapping, the new CoAP option and response codes presented in [5] have also been included, as explained in the next subsection.

\section{B. Enhancements for CoAP}

Based on the work presented in [4], the authors proposed enhancements for the subscriptions mechanisms of CoAP's observe in [5], aiming to reduce the overhead and latency. In this section, we first define the requirements for more advanced subscription mechanisms and then propose a solution.

- Subscribe with PUT/POST. A client can only subscribe with a GET request with the current Observe extension. Implementing this requirement allows to a client to create or update a resource and subscribe to it in a single step. 
- Response with no payload. In some use cases, a client might want to subscribe to get notifications from a resource (for example on low bandwidth data connections), but might not need the current representation when subscribing, or it may not have a valid value yet. This response can be heavy if the resource representation is big.

- Subscribe through related resources. Resources can be related to other resources, for instance configuration resources. A client might want to update, create or read these configuration resources and subscribe to the resources they are related to. With these requirements they can do this in a single step, while with the current mechanisms of CoAP and its Observe, the client would need to send two independent requests.

To address these requirements, our proposal is to create two new options and four new response codes. Fig. 2 shows the new proposed options while Fig. 3 shows the new proposed response codes.

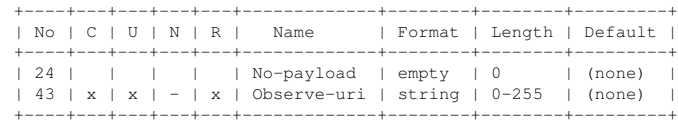

Fig. 2. New proposed options for CoAP.

The two new options are named No-payload and Observe-uri. The former allows to indicate to the server that the client is not interested in receiving the resource representation in that moment, while the latter indicates that the client wants to subscribe to the notifications of a related resource.

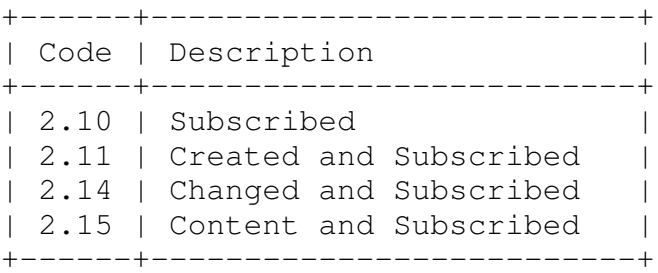

Fig. 3. New response codes for CoAP.

These response codes allow to the server to indicate the client that the subscription has been successful, but it does not include the resource representation because the client asked not to receive it.

These new CoAP option and response codes can address the requirements presented earlier and reduce the overhead and the number of messages when subscribing to resources. They are explained in more detail in the next paragraphs. This is especially relevant on low bandwidth data connections or dynamic environments, where devices connect and disconnect to and from the network, thus subscribe and unsubscribe often, such as Vehicle-to-Vehicle networks.

1) Subscribe with PUT/POST: Currently, clients can only subscribe using GET requests, not PUT or POST. We propose to use the Observe option also with PUT or POST requests, mimicking GET requests. This way, the interaction is similar with PUT and POST requests, where the server responds with
"2.01 Created" or a "2.04 Changed" code along with the Observe option. This way, the client can create or update a resource while subscribing to its notifications. After the response, the notifications work exactly as current notifications, "2.05 Content" and the observe option with the sequence number [37]. A resource update while subscribing, its response and two notifications are presented if Fig. 4.

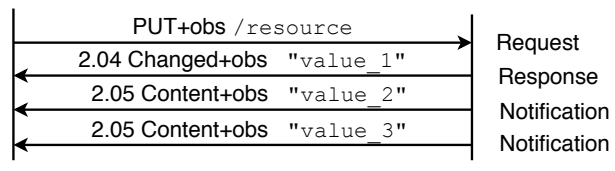

Fig. 4. Update the values of a resource, subscribe to it and get notifications.

Like a regular subscription with a GET request, if the request can be handled correctly but the subscription cannot be carried out, the response will not include the observe option.

2) Lightweight responses: The first new CoAP option we proposed is no-payload. With this option a client can let the server know that it does not need the resource representation when subscribing. As shown in Fig 2, following CoAP's convention to define the code numbers, we propose the identifier 24. It is not critical, the server will send the entire resource representation if it does not recognize the option. When including the no-payload option, the observe option must also be present in the request. To handle responses, new codes are presented, presented in Fig. 3, i.e. "2.10 Subscribed", "2.11 Created and Subscribed" and "2.14 Changed and Subscribed". These responses indicate that the client has been successfully subscribed but the response does not have a payload with the representation of the resource. The response code identifiers have been selected following a parallelism with previous response codes, from $2.0 \mathrm{X}$ codes to $2.1 \mathrm{X}$ codes. The similarities are the following, including a "2.15 Content and Subscribed" code explained in the next requirement:

- Subscribed: 2.10 .

- Created and Subscribed: 2.11, similar to 2.01 Created.

- Changed and Subscribed: 2.14, similar to 2.04 Changed.

- Content and Subscribed: 2.15, similar to 2.05 Content.

In Fig. 5 an interaction is shown, a GET request to subscribe to a resource with no interest on receiving the current state, i.e., with observe and no-payload options. The response for a correct interaction is "2.10 Subscribed", while "5.00 Internal Server Error" or "5.03 Service Unavailable" are the responses if the subscription fails, depending on MAX-AGE option being included or not. If the request cannot be satisfied for some other reason, regular CoAP error codes are to be used.

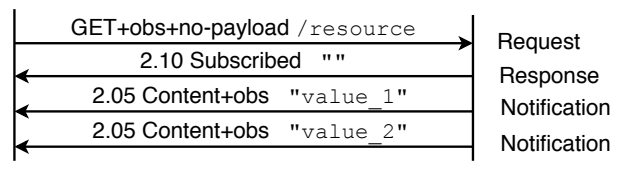

Fig. 5. Subscription to a resource without getting the current state and get notifications.

If a client wants to update a resource, for instance with a PUT request, the response will be a "2.01 Created" or 
"2.04 Changed" depending on whether the resource previously existed or not. Fig. 6 represents a request to update a resource and subscribe to it without wanting the current representation. Thus, the response is a " 2.14 Changed and Subscribed", meaning that the resource has been updated and the client has been subscribed. In the case of the resource not being present previously, the response would be "2.11 Created and Subscribed". POST requests work in the same way. If the resource is correctly created or updated but the subscription is not possible, the server will respond with " 2.01 Created" or "2.04 Changed". If there is any other problem, current regular response codes apply.

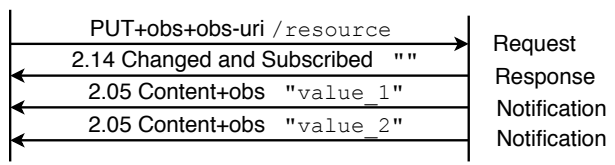

Fig. 6. Change a resource, subscribe to it but do not receive a payload, then get notifications.

3) Subscribe through other resources: When a resource has another related resource, a client might want to subscribe to one of them while sending a request to the other, e.g., a configuration resource. For that, we propose to use the code 43 for an option named observe-uri. This way, a resource, for instance a configuration resource, can be read, updated or created while subscribing to the main resource. This code is critical as the client is expecting to be subscribed to another resource, hence, if this cannot be fulfilled, the request should not be dealt with.

In Fig. 7 can be seen a GET request to a resource and a subscription to a different one. A "2.15 Content and Subscribed" response will include the representation of the resource the request has been sent to and the client will be subscribed to the related resource. If the subscription request cannot be handled, the response code falls back to " 2.05 Content". For the rest of the issues, usual error codes are used. However, when the resource the client wants to subscribe to does not exist, the correct response code is "4.02 Bad-Option" and the response should include the observe-uri option.

The "2.15 Content and Subscribed" response code includes the payload of the resource the request has been sent to. However, the notifications that will arrive after will have the resource representation the client has subscribed to, different resource from the first response.

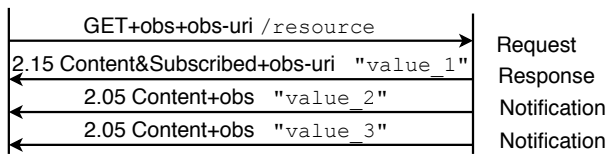

Fig. 7. Get the representation of a resource, subscribe to a different resource and get notifications.

In the case of PUT and POST request, the message exchange will be similar, with " 2.01 Created" responses if the resource did not previously exist or "2.04 Changed" if it did. Fig. 8 shows an example of a PUT request to a resource that already existed, thus, getting a "2.04 Changed" response. In the event that the resource has been correctly created or updated but the server cannot subscribe the client in the observers list, the response will not have the Observe option. If some other error occurs, current error codes should be used. If the related resource does not exist, the handling is the same as a GET request, a "4.02 Bad-Option" response and the observe-uri option.

\begin{tabular}{|c|l} 
PUT+obs+obs-uri /resource & $\begin{array}{l}\text { Request } \\
\text { Response } \\
\text { Notification }\end{array}$ \\
\cline { 1 - 1 } 2.04 Changed+obs+obs-uri "value_1" & Notification
\end{tabular}

Fig. 8. Update a resource, subscribe to a different resource and get notifications.

Observe-uri option and no-payload option can also be used in conjunction. Fig. 9 shows a client subscribing to a related resource and not needing the current state of the resource it is sending the request to. At first sight, this seems to have little sense, a client subscribing directly to the related resource through a GET request with the no-payload option present would have the same result. However, sending the request to a resource and subscribing to the related one a client can subscribe to a resource and at the same time, check if the related resource, e.g., a configuration resource already exists. The server will respond with a " 2.10 Subscribed" if the request can be correctly handled. In the event of an error, the server will behave as previously described, i.e., regular error codes for general errors, "4.02 Bad-Option" with observe-uri option if the related uri does not exist, and "5.00 Internal Server Error" or "5.03 Service Unavailable" if the client cannot be subscribed.

\begin{tabular}{|c|c|c|}
\hline \multicolumn{2}{|c|}{ GET+obs+no-payload+obs-uri / resource } & \multirow{4}{*}{$\begin{array}{l}\text { Request } \\
\text { Response } \\
\text { Notification }\end{array}$} \\
\hline 2.10 Subscribed + & obs-uri " " & \\
\hline 2.05 Content+obs & "value_1" & \\
\hline 2.05 Content+obs & "value_2" & \\
\hline
\end{tabular}

Fig. 9. Poll a resource without getting the representation, subscribe to a different resource and get notifications.

In case the client wants to create or update a resource through a PUT or POST request, the behaviour is similar to GET. The server will respond with "2.11 Created and Subscribed" or "2.14 Changed and Subscribed" response code with the observe-uri option present but with no payload. Fig. 10 shows a PUT request with successful subscription to a resource that already existed. If the subscription is not possible, the server will use "2.01 Created" or "2.04 Changed" codes. Same as a GET request, if the resource in the observeuri option does not exist, the server will respond with a "4.02 Bad Option" code and the observe-uri option. If any other error occurs, regular option and response codes are to be used.

From Fig. 4 to Fig. 10, all the message exchanges show that the new option and response codes described here only matter in the subscription mechanisms, and the notifications are delivered as the current Observe describes.

For selecting the codes, we followed RFC 7252's rules, proposing code number 24 for "no-payload" and 43 for 


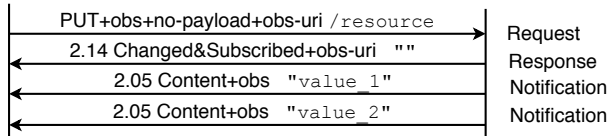

Fig. 10. Update a resource without getting the representation, subscribe to a different resource and get notifications.

"observe-uri". "No-payload" is elective (non-critical) as if ignored, the server can handle the request sending the representation of the resource. For that reason, it is also safe to forward through a proxy and it is not repeatable. The option just indicates if the client wants a payload on the response or not, hence, it has no length nor format. "Observe-uri" is parallel to "uri-path", indicating to which resource the client wants to subscribe. Therefore, it is a critical, an unsafe to forward and a repeatable option. Similar to the "uri-path" option, it is represented in string format and has a maximum length of 255. This option indicates the relative path from the resource from "uri-path", therefore, ".." is accepted. One condition to subscribe to the resource of "observe-uri" is that the resource has to be observable.

\section{IMPLEMENTATION}

In order to evaluate the proposed mechanism, we have extended the software tool developed and first introduced in [38]. With this tool, a system architect/designer is able to create an IEC 61850 compatible data model graphically (by means of an Ecore meta model and an Eclipse Plugin constructed based on it [39]). Given this information, the tool generates the source code for HTTP or WS-SOAP communication protocols (whichever the system architect/designer selected). Then, this generated code integrates the data model with the communication stack selected by the user, following the services model of the IEC 61850. A screenshot of this tool is shown in Fig 11.

For the present work, a new protocol has been added to the choice, i.e., CoAP. In [3], a mapping of the IEC 61850 to CoAP was proposed, and later validated in [4] with some adaptations. The HTTP mapping uses JSON as the representation format for the data, while WS-SOAP uses XML. For the validation of the mapping, JSON was used for the data representation. In this work, we complete the entire IoT stack and change the resource representation of the work presented in [4] to CBOR in the case of the CoAP mapping.

In addition, in [5], the authors propose new CoAP option and response codes to enhance the subscription mechanisms as explained in Section IV. In the present work, we add those options (in the SetBRCBValues and SetURCBValues functions) to the system, as shown in Table II. When a client wants to subscribe to the reports when updating a BRCB or URCB, with the options and response codes of the current specifications of CoAP, it needs to first update the values, and then send another request to subscribe to the reports. With our new options, this can be done in a single step. If the client just wants to update the values, but not to subscribe to the Reports, the request is the same on the old and new proposals. Hence, our proposal supports the following requirements:

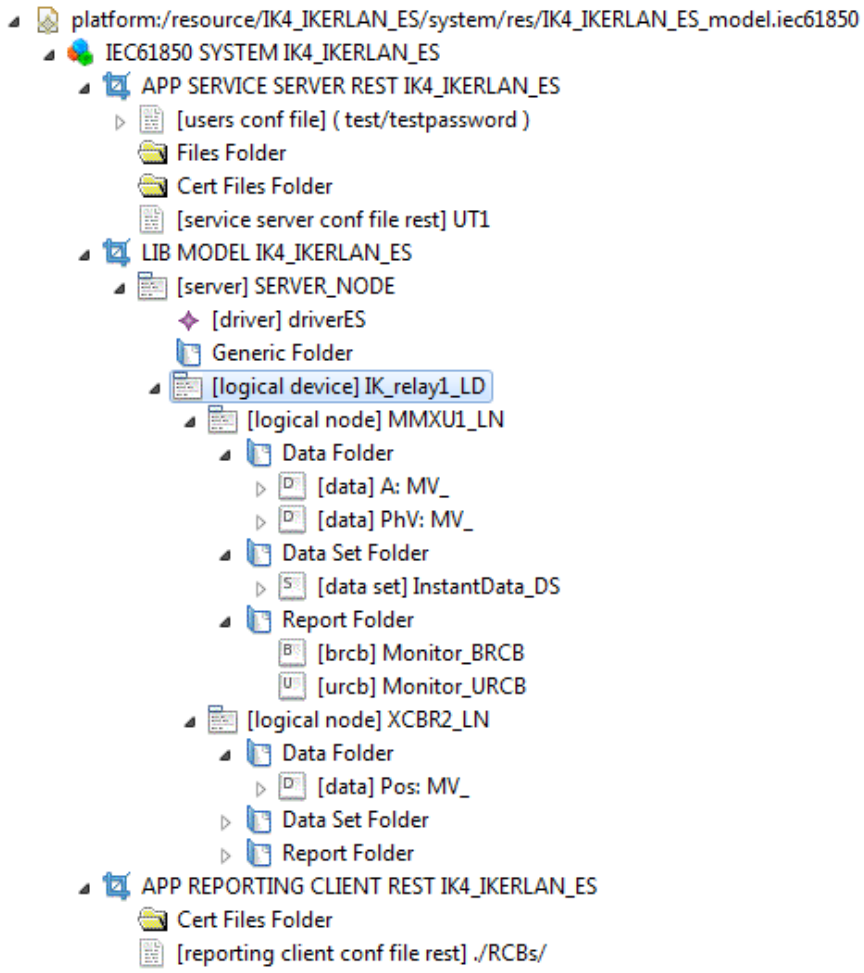

Fig. 11. Screenshot of the IEC 61850 tool.

- Subscription to the reports with the no-payload option activated, thus not requesting the report before it is generated.

- Subscription to the reports through the $[\mathrm{UB}] \mathrm{RCB}$ resource, instead of directly.

- Subscription when updating the [UB]RCB resource, i.e., with a PUT request.

TABLE II

UPDATING [UB]RCBS AND SUBSCRIBING TO REPORTS WITH THE CURRENT COAP SPECIFICATION AND WITH THE NEW ENHANCED SUBSCRIPTION MECHANISM.

\begin{tabular}{|c|c|c|}
\hline \multicolumn{3}{|c|}{ Current CoAP } \\
\hline SetBRCBValues & coap: $/ /\{$ host $\} / \mathrm{LDs} /\{\mathrm{LDs}\} /\{\mathrm{LN}\} /\{$ BRCBs $\} / \mathrm{BRCB}$ & PUT \\
\hline Subscribe & coap: $/ /\{$ host $\} /$ LDs $/\{$ LDs $\} /\{$ LN $\} /\{$ BRCBs $\} /$ BRCB $/$ Reports & GET+Obs \\
\hline SetURCBValues & coap: $/ /\{$ host $\} /$ LDs $/\{$ LDs $\} /\{$ LN $\} /\{$ URCBs $\} /$ URCB & PUT \\
\hline Subscribe & coap: $/ /\{$ host $\} /$ LDs $/\{$ LDs $\} /\{$ LN $\} /\{$ URCBs $\} /$ URCB/Report & GET+Obs \\
\hline \multicolumn{3}{|c|}{ New CoAP option and response codes } \\
\hline SetBRCBValues & $\begin{array}{l}\text { coap://\{host }\} / \text { LDs } /\{\text { LDs }\} /\{\text { LN }\} /\{\text { BRCBs }\} / B R C B \\
\text { observe-uri: Reports }\end{array}$ & $\begin{array}{l}\text { PUT+obs+no-payload+ } \\
\text { observe-uri }\end{array}$ \\
\hline SetURCBValues & $\begin{array}{l}\text { coap: } / /\{\text { host }\} / L D s /\{\text { LDs }\} /\{\text { LN }\} /\{\text { URCBs }\} / \text { URCB } \\
\text { observe-uri: Report }\end{array}$ & $\begin{array}{l}\text { PUT+obs+no-payload+ } \\
\text { observe-uri }\end{array}$ \\
\hline
\end{tabular}

The IEC 61850 tool generates the code structured in different layers (see Fig. 12). At the bottom layer, lib-model-kernel includes the core functions of the IEC 61850. On top of that is lib-model-specific, which represents the data model created with the Eclipse plugin tool, that is to say, the domain specific data model. For this work, the data model used for the validation is a model of an electrical substation, see Fig. 11. The libraries used to create the servers for the different communication protocols are 


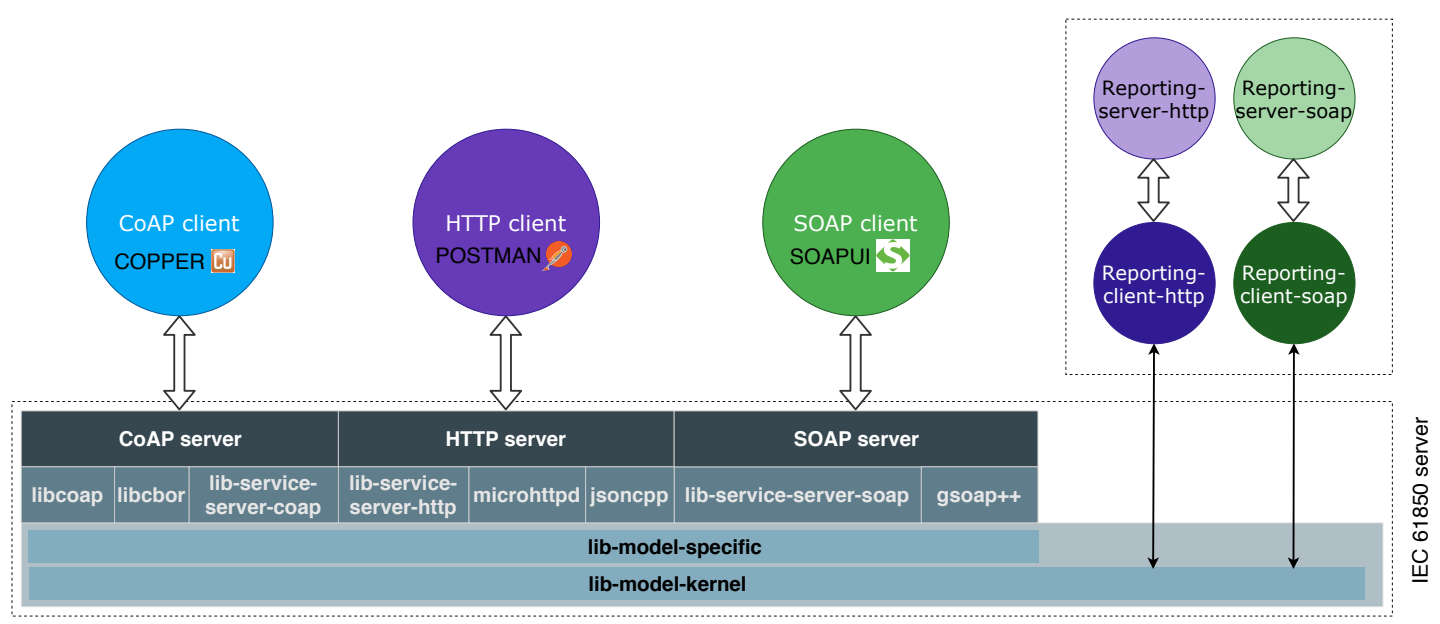

Fig. 12. Layered overview of the reference design of the IEC 61850 software tool.

situated on the next layer ${ }^{6}$. Each of them has its helper library for the servers (libcoap [40] ${ }^{7}$, microhttpd [42] and gsoap++ [43]) and the representation format of the data (libcbor [44], jsoncpp [45]). Finally, in the last layer, we can find the generated servers, which integrate the libraries.

In HTTP and WS-SOAP, the reporting services are more complex. As these protocols do not allow to push notifications, new servers and clients have to be developed and deployed: the servers store reports in a folder on the system. Reporting clients watch these folders for new reports, and request new reports from the reporting server when they detect them. CoAP can provide this functionality more gracefully, thanks to its Observe extension. With this extension, the same client can subscribe to notifications and request push notifications from the server that contains generated reports.

Based on the extended tool and the representation of the data model for an electrical substation, we already have the necessary basis to carry out the evaluation. The artifacts generated by the tool provide the common framework for carrying out a comparison between different communication protocols.

\section{Evaluation}

The main goal of our evaluation is to demonstrate that it is possible to integrate IEC 61850 with an IoT communication protocol. Thus, in this section, we evaluate the performance of our system and compare it against WS-SOAP and HTTPbased systems. For comparison purposes, we have selected two metrics: the response time, i.e., the time that the client waits from when it sends the request to when it receives the response with the requested data or acknowledgment; and the required number of bytes to send the data, taking into account the payload, overhead and the total bytes sent. The analysis of the bytes needed for the communication is especially relevant in constrained, low-bandwidth environments.

\footnotetext{
${ }^{6}$ These libraries are lib-service-server-http, lib-service-server-soap and lib-service-server-coap to create the HTTP, WS-SOAP and CoAP servers, respectively.

${ }^{7}$ Among the available CoAP implementations [41], we selected the develop branch version of Feb. 5, 2018 of libcoap.
}

For the server part, instead of using proprietary hardware (with a proprietary operating system), we have decided to use an openly available board, i.e., a Raspberry Pi 3 [46]. ${ }^{8}$ Raspberry Pi boards have been used in industrial environments for prototype and lab testing, and are currently expanding to real-world industrial deployments as well ${ }^{9}$.

Our clients run on a Windows 10 PC; the selected HTTP client is Postman 6.0.10 $0^{10}$, we used SoapUI 5.4.0 $0^{11}$ for WSSOAP and Copper 1.0.1 ${ }^{12}$ on top of Firefox 46.0 for CoAP. The clients have been connected to the servers via Wi-Fi and the client PC runs Wireshark ${ }^{13}$ to analyze the network traffic and measure overheads and response times. As stated in the introduction, wireless technologies (including cellular technologies) are more and more spreading into industrial deployments [47], especially for remote settings (e.g., electrical substations in remote environments) due to lower installation cost, sufficient data rates, good reliability and ease of deployment. Whereas the majority of substations feature Ethernet or power line networks [48], deploying wired networks requires more effort in time and economic cost. Overall, IEEE 802.11 protocols are suitable for ensuring high security and QoS for less critical smart distribution network applications [49]. Hence, we expect a transition to Wireless-based substations or mixed approaches, where interoperability is ensured through standards such as IEC 61850.

\section{A. Response Time}

We start by evaluating the performance of the different approaches in terms of response time. To do that, the maximum and median of a series of 500 requests are presented in Table III. Some of the functions presented in Table I have been

\footnotetext{
${ }^{8}$ Our Raspberry Pi 3 device features a Quad-Core ARM Cortex A53 CPU running at $1.2 \mathrm{Ghz}$ and $1 \mathrm{~GB}$ of RAM; a $16 \mathrm{~GB}$ class 10 microSD card hosts the Raspbian Stretch operating system.

${ }^{9}$ https://www.rs-online.com/designspark/raspberry-pi-and-arduino-inindustrial-environments

${ }^{10} \mathrm{https}: / /$ www.getpostman.com/

${ }^{11} \mathrm{https} / / /$ www.soapui.org/

${ }^{12}$ https://github.com/mkovatsc/Copper

${ }^{13}$ https://www.wireshark.org/
} 
left out of this analysis: Reporting subscription (functions 21 and 26) and Report (functions 17, 18, 22 and 23) have not been included. This is because they work in a different way in the WS-SOAP and HTTP implementations, as explained in Section IV-A: they poll the server to check for existing reports and then request them. In CoAP's case, CON messages have been used in this evaluation. However, the performance results with NON messages would be similar, as the responses are piggybacked on acknowledgements for CON messages.

TABLE III

MAXIMUM AND MEDIAN OF THE RESPONSE TIMES IN MS WITH COAP, HTTP AND SOAP.

\begin{tabular}{r|r|r|r|r|r|r}
\multirow{2}{*}{ Id } & \multicolumn{3}{|c|}{ Maximum latency $(\mathrm{ms})$} & \multicolumn{3}{|c}{ Median latency $(\mathrm{ms})$} \\
\cline { 2 - 7 } & CoAP & HTTP & SOAP & CoAP & HTTP & \multicolumn{1}{c}{ SOAP } \\
\hline 1 & 21.8 & 32.3 & 12.7 & 5.7 & 5.4 & 5.1 \\
2 & 20.8 & 69.7 & 38.2 & 5.8 & 7.6 & 5.8 \\
3 & 37.3 & 31.7 & 212.4 & 5.9 & 7.7 & 8.0 \\
4 & 36.6 & 76.8 & 20.7 & 6.6 & 8.2 & 6.9 \\
5 & 11.9 & 226.6 & 65.4 & 6.2 & 5.8 & 9.1 \\
\hline 6 & 29.9 & 36.1 & 103.2 & 6.1 & 7.1 & 8.8 \\
7 & 62.6 & 82.0 & 97.7 & 6.0 & 5.2 & 7.0 \\
8 & 5119.1 & 484.9 & 297.4 & $\mathbf{3 1 2 7 . 9}$ & 64.2 & 75.5 \\
9 & 6495.6 & 312.5 & 304.1 & $\mathbf{2 7 2 2 . 7}$ & 256.2 & 73.5 \\
10 & 41.6 & 27.7 & 25.0 & 7.4 & 7.2 & 8.8 \\
\hline 11 & 10.0 & 155.5 & 42.3 & $\mathbf{5 . 9}$ & 10.0 & 11.6 \\
12 & 18.6 & 299.5 & 77.6 & 6.1 & 209.6 & 8.7 \\
13 & 14.5 & 93.0 & 28.3 & 6.0 & 10.5 & 7.7 \\
14 & 325.4 & 102.9 & 31.6 & $\mathbf{2 3 2 . 5}$ & 13.1 & 13.7 \\
15 & 92.0 & 46.7 & 220.8 & 5.9 & 6.3 & 9.1 \\
\hline 16 & 16.7 & 94.6 & 23.8 & 6.4 & 7.4 & 6.8 \\
19 & 37.7 & 42.4 & 25.2 & 6.2 & 8.5 & 8.6 \\
20 & 81.3 & 677.7 & 76.4 & $\mathbf{5 . 5}$ & 209.3 & 9.5 \\
24 & 7.6 & 245.0 & 24.3 & $\mathbf{6 . 2}$ & 209.5 & 9.9 \\
25 & 14.0 & 103.4 & 32.9 & 5.4 & 7.7 & 7.8 \\
\hline 27 & 19.6 & 103.1 & 65.0 & 9.7 & 8.3 & 5.4 \\
28 & 67.7 & 97.8 & 23.5 & 8.0 & 6.2 & 5.1 \\
29 & 61.7 & 14.5 & 23.5 & 7.1 & 5.5 & 5.1 \\
30 & 1018.6 & 272.9 & 83.9 & $\mathbf{6 8 2 . 3}$ & 219.3 & 19.7 \\
31 & 14.8 & 26.5 & 308.3 & 6.0 & 6.5 & 9.2 \\
\hline 32 & 76.5 & 262.8 & 66.8 & 6.0 & 209.9 & 8.9 \\
33 & 15.4 & 227.4 & 32.4 & 6.1 & 207.6 & 8.6 \\
34 & 18.1 & 157.3 & 32.8 & 5.6 & 8.8 & 8.4 \\
35 & 13.4 & 40.6 & 17.6 & 5.8 & 9.1 & 7.1 \\
& & & & & &
\end{tabular}

The left three columns of Table III show the maximum values of the response times while the three columns on the right present the median values. We selected the maximum time as a representation of a worst case scenario, but the most important metric is the median. In our evaluation, the median typically shows better values than the average, as it mitigates outliers. Analyzing the median values, CoAP has the lowest, with values between $5-8 \mathrm{~ms}$ in most cases. Calculating the average of the response times of the mapped functions, CoAP's response time is 71\% of HTTP's and $85 \%$ of WS-SOAP's, where we do not count the functions with block-wise transfer (functions 8, 9, 14 and 30). We do not include these functions because the block-wise transfer in CoAP works following the Stop \& Wait model, which increases response times by a large amount. Fig. 13 plots the ratio of median response time values of the functions. CoAP is used as the baseline (at 100\%) and
HTTP and WS-SOAP data are shown related to that baseline (percentage $\left.=\frac{\text { CoAP median }}{\text { Protocol median }} \times 100\right)$. In the chart, values below $100 \%$ mean that CoAP is faster than the compared protocol; this is the case for most functions.

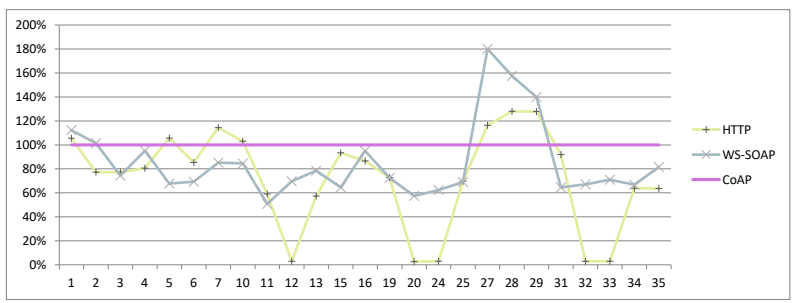

Fig. 13. Percentage of CoAP's median response time compared to HTTP and WS-SOAP in the functions without block-wise transfer. Values below $100 \%$ mean that the protocol is slower than CoAP.

On the other hand, we observe negative peaks for several functions, bold in Table III, with median values up to 2 or 3 seconds (functions 8 and 9). This occurs when CoAP's blockwise transfer extension is required: CoAP limits the size of the payload to 1024 bytes (the entire message must fit in a single MTU) - whenever the payload is larger than that size, it needs to be chunked and sent in different messages, with the entire header and using a Stop \& Wait method for successive parts (i.e., each chunk needs to be acknowledged before requesting the next). In those cases, the underlying TCP's sliding window approach (i.e., new packets can be sent before receiving all acknowledgements of previous packets) that underpins HTTP and WS-SOAP outperforms CoAP.

\section{B. Overhead}

The last aspect of the evaluation is to compare the overhead of the messages needed to request, change or delete data from a server. Fig. 14 (bars) compares payload and overhead. In the overhead, we count the bytes added by the different network protocols to send the data, i.e., the bytes on the wire minus the payloads. For these measurements, TCP's handshake to open and close the channel and the keep-alive messages to maintain the channel open have not been counted. The functions for subscribing to the reports (functions 21 and 26) have been left out because they are not part of the standard and as previously mentioned WS-SOAP and HTTP do not subscribe to reports. In the WS-SOAP implementation, a single function called Logoff substitutes the Release and Abort functions, thus, data is the same for both. Bars represent the number of bytes for each protocol and resource representation (payload in CBOR, JSON or XML for CoAP, HTTP and SOAP respectively) need to send the data from the server to the client.

This comparison clearly highlights the differences between data representation formats, especially in the case of XML (used in WS-SOAP), which is the largest by far. The lines on Fig. 14 represent the relation of the representation of the resources compared to CoAP. Fig. 15 contains the same data, but focuses on comparing HTTP (JSON) and CoAP (CBOR), as the representation of WS-SOAP (XML) is much bigger, going as high as $17500 \%$. JSON (HTTP) performs slightly worse than $\mathrm{CBOR}$ (used in CoAP); on average, CBOR requires 


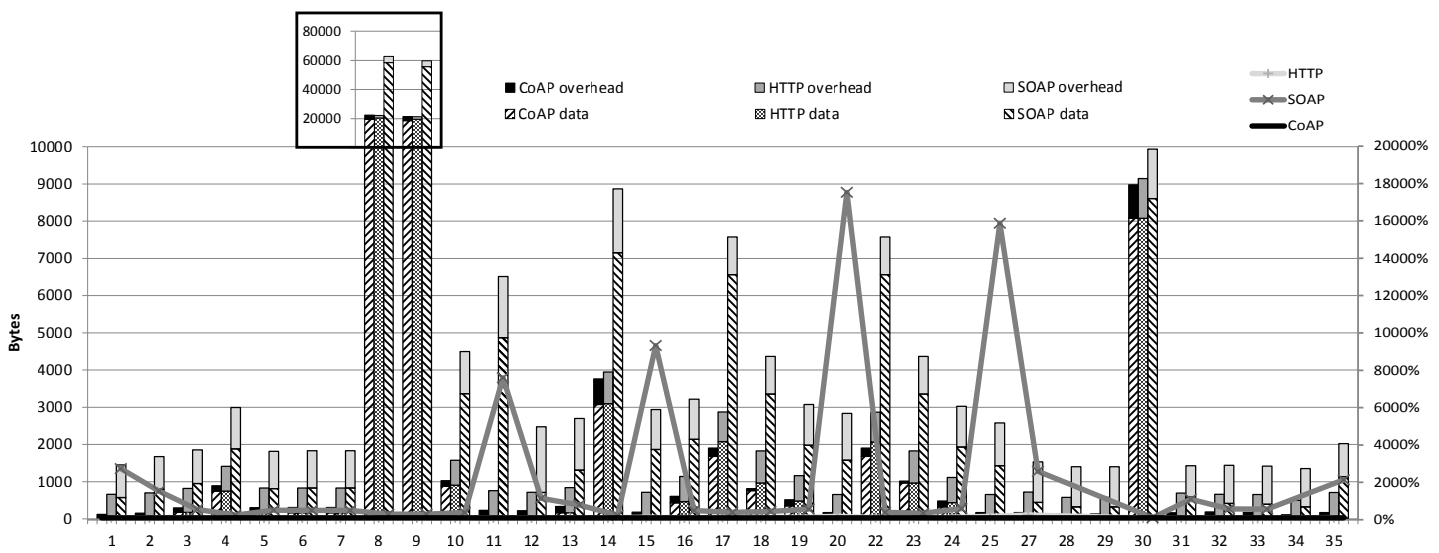

Fig. 14. The bars show the number of bytes required for the resource representation and overhead in CoAP, HTTP and WS-SOAP implementations. The lines represent the overheads of HTTP and WS-SOAP as fractions of the CoAP baseline.

$89 \%$ of the bytes on average for all the analyzed functions compared to JSON, and $19 \%$ compared to XML.

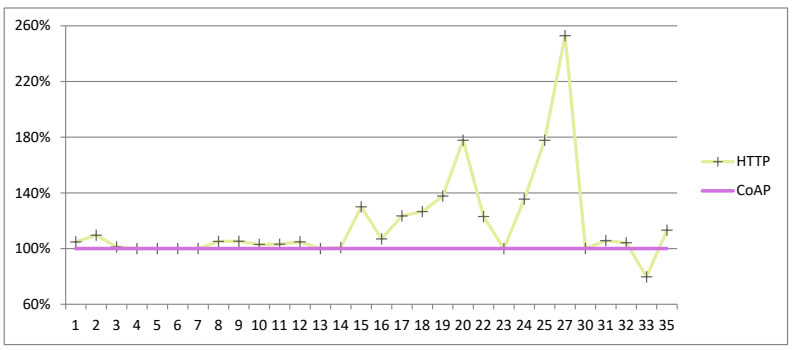

Fig. 15. Percentage of required bytes for the resource representation of HTTP's (JSON) implementation with CoAP's (CBOR) as baseline.

In terms of overhead, WS-SOAP has the largest because it is a very verbose protocol. HTTP has lower overhead, but CoAP has the lowest of the three. However, in the cases of big payloads, when the block-wise transfer extension is needed, CoAP can have more overhead than HTTP because each request includes the entire header, while HTTP chunks the entire message, with a single header for all. Fig. 16 shows the comparison of the total number of bytes sent, i.e., payload and overhead, using CoAP's implementation as baseline. $\mathrm{CoAP}+\mathrm{CBOR}$ uses on average $44 \%$ of the bytes comparing to HTTP+JSON and 18\% comparing to WS-SOAP+XML.

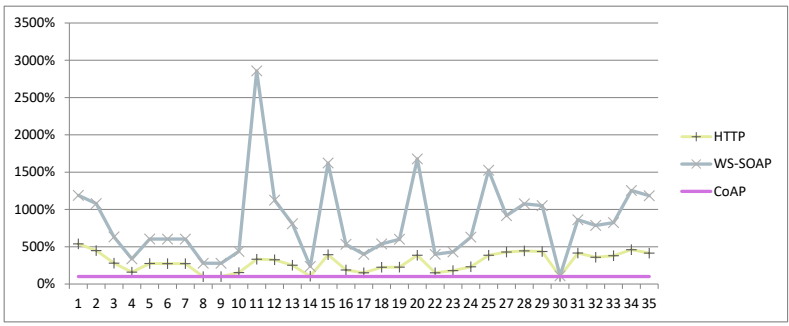

Fig. 16. Percentage of total number of required bytes (payload + protocol overhead) for sending data in HTTP's and WS-SOAP's implementation with CoAP's as baseline.

The mapping has been updated to include the options proposed in [5], as shown in Table II. Fig. 17 presents the difference in the number of bytes needed to update a BRCB or an URCB and get the reports when generated. Two cases have been considered, large and small reports. The left bar of each group represent the current message exchange, including the resource update and the subscription messages. The right bar represent the message exchange with the new options.

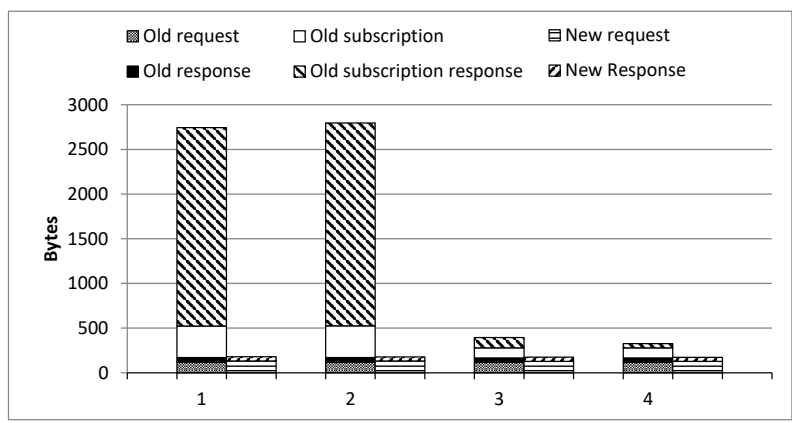

Fig. 17. Overhead in the old and new approaches: SetBRCBValues (1) and SetURCBValues (2) with a big payload; and SetBRCBValues (3) and SetURCBValues with small payload (4). The left bar of each pair represents the current message exchange with CoAP. The right bar, the new approach with new option and response codes.

To conclude the previously described evaluation, we have shown that CoAP can be used for communication with IEC 61850 thanks to its combination of client-server and publish/subscribe paradigms. This makes it more straightforward to use for reporting functions, compared to HTTP and WSSOAP, which need to poll for reports. In addition, the protocol overhead, the size of the resource representation and the response time are lower in most cases. However, it is important to note that CoAP is outperformed by the other protocols when it switches to block-wise transfer for sending large payloads.

\section{DISCUSSION AND CONCLUSION}

In this paper, we have presented an evaluation of the performance of the IEC 61850 standard over the CoAP communication protocol and compared it to implementations based on HTTP and WS-SOAP.

First, we demostrate that it is possible to integrate IEC 61850 with an IoT protocol stack, using CoAP and CBOR. 
Through our experiments, we verify that CBOR representation requires fewer bytes than JSON and XML, and that CoAP requires fewer bytes on the network than the HTTP and WS-SOAP. This also entails lower response time for the CoAP+CBOR option when block-wise transfer is not used.

Second, we have validated that the limitations mentioned in [4] regarding the subscription mechanism can be overcame with the newly proposed options and response codes, leading to less bandwidth usage. The standard Observe extension requires extra messages and bytes for use cases involving the IEC 61850 among others. With our proposed extensions for CoAP Observe, we claim that interacting with the server is more natural and network overhead significantly lower.

However, for large payloads, the block-wise transfer in CoAP adds a lot of overhead when many blocks are required. The first future line of work arises from this observation: achieving a more effective block-wise transfer interaction to send large payloads in CoAP. The current block-wise mode sends the chunks one by one through the Stop \& Wait paradigm, with the entire header for each block.

Another line of research from this work is to conduct the evaluation on a simulation tool (e.g. Omnet++, Ns-2, etc.) or a real hardware in an electrical substation. This work has been deployed on a lab prototype over a dedicated WiFi network and point-to-point communication, with no interference nor packet loss. Hence, the next step is to create a simulation with several nodes and a low-bandwidth network that might be congested, preparing for a deployment of the system in a real environment. This way, we could test the performance in environments closer to harsher industrial deployments.

Regarding the interoperability of IEC 61850, we consider that the model itself and the CoAP+CBOR mapping could also be applied to other DERs, such as electric vehicles, microgrids, photovoltaics, microturbines, etc. - in this way, we would enable advanced properties such as real-time data analysis, proactive control, and automated outage mitigation mechanisms [50]. Furthermore, IEC 61850 might also be applicable in other domains that are relevant in the SG context (e.g., Smart Elevators, Catenary-free Trams or Wind Turbines [38]) while other domains to not lend themselves to this or similar standards (e.g., Smart Homes, Ambient Media and Health management, etc. [14]).

\section{REFERENCES}

[1] K. Diwold, S. Mayer, A. Einfalt, J. X. Parreira, J. Hodges, D. Anicic, and R. Mosshammer, "Grid Watch Dog: A Stream Reasoning Approach for Lightweight SCADA Functionality in Low-Voltage Grids," in Proceedings of the Eighth International Conference on the Internet of Things (IoT 2018), 2018.

[2] S. Nwabuona, M. Schuss, S. Mayer, K. Diwold, L. Krammer, and A. Einfalt, "Time-synchronized Data Collection in Smart Grids through IPv6 over BLE," in Proceedings of the Eighth International Conference on the Internet of Things (IoT 2018), 2018.

[3] M. Iglesias-Urkia, A. Urbieta, J. Parra, and D. Casado-Mansilla, "IEC 61850 Meets CoAP: Towards the Integration of Smart Grids and IoT Standards," in Proceedings of the Seventh International Conference on the Internet of Things (IoT 2017). New York, NY, USA: ACM, 2017, pp. 3:1-3:9.

[4] M. Iglesias-Urkia, D. Casado-Mansilla, S. Mayer, and A. Urbieta, "Validation of a CoAP to IEC 61850 Mapping and Benchmarking vs HTTP-REST and WS-SOAP," in IEEE International Conference on Emerging Technologies and Factory Automation, ETFA, vol. 2018September, 2018, pp. 1015-1022.

[5] - "Enhanced Publish/Subscribe in CoAP: Describing Advanced Subscription Mechanisms for the Observe Extension," in Proceedings of the Eighth International Conference on the Internet of Things (IoT 2018), 2018.

[6] B. Petersen, H. Bindner, S. You, and B. Poulsen, "Smart Grid Communication Middleware Comparison," SmartGreens, Porto, 2017.

[7] M. Albano, L. L. Ferreira, L. M. Pinho, and A. R. Alkhawaja, "Messageoriented middleware for smart grids," Computer Standards \& Interfaces, vol. 38, pp. 133-143, Feb 2015.

[8] IEC TC-88, "Wind Energy Generation Systems - Part 25-4: Communications for Monitoring and Control of Wind Power Plants - Mapping to Communication Profile," 2016.

[9] R. Sanz, J. A. Clavijo, M. J. Segarra, A. de Antonio, and M. Alonso, "CORBA-Based Substation Automation Systems," in Proceedings of IEEE Conference on Control Applications, 2001.

[10] I. Calvo, O. García De Albéniz, A. Noguero, and F. Pérez, "Towards a Modular and Scalable Design for the Communications of Electrical Protection Relays," IECON Proceedings (Industrial Electronics Conference), pp. 2511-2516, 2009.

[11] I. Calvo, O. Garcia de Albéniz, and F. Pérez, "A Communication Backbone for Substation Automation Systems Based on the OMG DDS Standard," in Przeglad Elektrotechniczny, vol. 88, no. 1 A, 2012, pp. 146-150.

[12] Y. Bi, L. Jiang, X. J. Wang, and L. Z. Cui, "Mapping of IEC 61850 to Data Distribute Service for Digital Substation Communication," in IEEE Power and Energy Society General Meeting, 2013, pp. 1-5.

[13] A. B. Pedersen, E. B. Hauksson, P. B. Andersen, B. Poulsen, C. Traeholt, and D. Gantenbein, "Facilitating a generic communication interface to distributed energy resources: Mapping iec 61850 to restful services." IEEE, Oct 2010, pp. 61-66.

[14] J. Parra, "Restful Framework for Collaborative Internet of Things Based on IEC 61850," Ph.D. dissertation, Universidad del País Vasco - Euskal Herriko Unibertsitatea (UPV/EHU), 2016.

[15] S. M. S. Hussain, M. A. Aftab, and I. Ali, "IEC 61850 Modeling of DSTATCOM and XMPP Communication for Reactive Power Management in Microgrids," IEEE Systems Journal, pp. 1-11, 2018.

[16] I.-J. Shin, B.-K. Song, and D.-S. Eom, "International Electronical Committee (IEC) 61850 Mapping with Constrained Application Protocol (CoAP) in Smart Grids Based European Telecommunications Standard Institute Machine-to-Machine (M2M) Environment," Energies, vol. 10, no. 3, p. 393, Mar. 2017.

[17] IETF, "Constrained RESTful Environments (core)," 2018, https:// datatracker.ietf.org/wg/core/documents.

[18] Z. Shelby, M. Vial, M. Koster, C. Groves, J. Zhu, and B. Silverajan, "Dynamic Resource Linking for Constrained RESTful Environments," Internet Engineering Task Force, Internet-Draft draft-ietf-core-dynlink05, Mar. 2018, work in Progress.

[19] M. Koster, A. Keränen, and J. Jimenez, "Publish-Subscribe Broker for the Constrained Application Protocol (CoAP)," Internet Engineering Task Force, Internet-Draft draft-ietf-core-coap-pubsub-04, Mar. 2018, work in Progress.

[20] G. Ketema, J. Hoebeke, I. Moerman, P. Demeester, L. S. Tao, and A. J. Jara, "Efficiently Observing Internet of Things Resources," in Proceedings - 2012 IEEE Int. Conf. on Green Computing and Communications, GreenCom 2012, Conf. on Internet of Things, iThings 2012 and Conf. on Cyber, Physical and Social Computing, CPSCom 2012. IEEE, 2012, pp. 446-449.

[21] R. Mietz, P. Abraham, and K. Römer, "High-level States with CoAP: Giving Meaning to Raw Sensor Values to Support IoT Applications," in 2014 IEEE Ninth International Conference on Intelligent Sensors, Sensor Networks and Information Processing (ISSNIP). IEEE, April 2014, pp. 1-6.

[22] G. Tanganelli, C. Vallati, E. Mingozzi, and M. Kovatsch, "Efficient Proxying of CoAP Observe with Quality of Service Support," in 2016 IEEE 3rd World Forum on Internet of Things, WF-IoT 2016. IEEE, 2017, pp. 401-406.

[23] A. Ludovici, E. Garcia, X. Gimeno, and A. C. Augé, "Adding QoS Support for Timeliness to the Observe Extension of CoAP," in International Conference on Wireless and Mobile Computing, Networking and Communications. IEEE, 2012, pp. 195-202.

[24] D. Sacramento, G. Schutz, and N. Correia, "Aggregation and Scheduling in CoAP/Observe Based Wireless Sensor Networks," in IEEE International Conference on Communications, vol. 2015-September. IEEE, 2015 , pp. 654-660. 
[25] N. Correia, G. Schutz, and A. Barradas, "Fairness for CoAP/Observe based wireless sensor networks with aggregation deployment." IEEE, Dec 2015, pp. 110-115.

[26] N. Correia, D. Sacramento, and G. Schutz, "Dynamic Aggregation and Scheduling in CoAP/Observe-Based Wireless Sensor Networks," IEEE Internet of Things Journal, vol. 3, no. 6, pp. 923-936, Dec. 2016.

[27] I. Ishaq, J. Hoebeke, I. Moerman, and P. Demeester, "Observing CoAP Groups Efficiently," Ad Hoc Networks, vol. 37, pp. 368-388, Feb. 2016.

[28] D.-K. Choi, J.-H. Jung, H.-W. Kang, and S.-J. Koh, "Cluster-based CoAP for Message Queueing in Intemet-of-Things Networks," in International Conference on Advanced Communication Technology, ICACT. IEEE, 2017, pp. 584-588.

[29] IEC TC-57, "Communication Networks and Systems in Substations Part 7-2: Basic Communication Structure for Substation and Feeder Equipment - Abstract Communication Service Interface (ACSI)," 2003.

[30] N. B. Priyantha, A. Kansal, M. Goraczko, and F. Zhao, "Tiny web services, design and implementation of interoperable and evolvable sensor networks." ACM Press, 2008, p. 253.

[31] Z. Shelby, K. Hartke, and C. Bormann, "The Constrained Application Protocol (CoAP)," RFC 7252, Jun. 2014.

[32] C. Bormann and P. E. Hoffman, "Concise Binary Object Representation (CBOR)," RFC 7049, Oct. 2013.

[33] IoT One, "Emerging Open And Standard Protocol Stack For IoT," 2016, https://www.iotone.com/guide/ emerging-open-and-standard-protocol-stack-for-iot/g494.

[34] B. Petersen, H. Bindner, S. You, and B. Poulsen, "Smart grid serialization comparison: Comparision of serialization for distributed control in the context of the Internet of Things." IEEE, Jul 2017, pp. 1339-1346.

[35] K. Li, A. Rahman, and C. Bormann, "Representing Constrained RESTful Environments (CoRE) Link Format in JSON and CBOR," Internet Engineering Task Force, Internet-Draft draft-ietf-core-links-json-10, Feb. 2018, work in Progress.

[36] M. Iglesias-Urkia, A. Orive, M. Barcelo, A. Moran, J. Bilbao, and A. Urbieta, "Towards a Lightweight Protocol for Industry 4.0: An Implementation Based Benchmark," in Proceedings of the 2017 IEEE International Workshop of Electronics, Control, Measurement, Signals and their Application to Mechatronics, ECMSM 2017, 2017.

[37] K. Hartke, "Observing Resources in the Constrained Application Protocol (CoAP)," RFC 7641, Sep. 2015.

[38] A. Iglesias, M. Iglesias-Urkia, B. López-Davalillo, S. Charramendieta, and A. Urbieta, "TRILATERAL: Software Product Line based Multidomain IoT Artifact Generation for Industrial CPS," in Proceedings of the 7th International Conference on Model-Driven Engineering and Software Development (MODELSWARD 2019), 2019, pp. 64-73.

[39] D. Steinberg, F. Budinsky, E. Merks, and M. Paternostro, EMF: Eclipse Modeling Framework. Pearson Education, 2008.

[40] O. Bergmann, "libcoap: C-Implementation of CoAP," 2017, https:// libcoap.net.

[41] M. Iglesias-Urkia, A. Orive, A. Urbieta, and D. Casado-Mansilla "Analysis of CoAP Implementations for Industrial Internet of Things: a Survey," Journal of Ambient Intelligence and Humanized Computing, Mar 2018

[42] Free Software Foundation, "GNU Libmicrohttpd," 2018, https://www. gnu.org/software/libmicrohttpd/.

[43] R. van Engelen, "gSOAP 2.8.66 User Guide," 2018, https://www.genivia. $\mathrm{com} / \mathrm{doc} / \mathrm{soapdoc} 2 . \mathrm{html}$

[44] P. Kalvoda, "CBOR format implementation for C \& others," 2017, http: //libcbor.org/.

[45] open-source-parsers, “JsonCpp," 2018, https://github.com/ open-source-parsers/jsoncpp.

[46] Raspberry Pi Foundation, "Raspberry Pi," 2018, https: //www.raspberrypi.org/.

[47] C. Kalalas, "Cellular networks for smart grid communication," Ph.D dissertation, Universitat Politècnica de Catalunya, 2018.

[48] D. D. Giustina, P. Ferrari, A. Flammini, S. Rinaldi, and E. Sisinni, "Automation of Distribution Grids With IEC 61850: A First Approach Using Broadband Power Line Communication," IEEE Transactions on Instrumentation and Measurement, vol. 62, no. 9, pp. 2372-2383, Sep. 2013.

[49] P. P. Parikh, T. S. Sidhu, and A. Shami, "A Comprehensive Investigation of Wireless LAN for IEC 61850-Based Smart Distribution Substation Applications," IEEE Transactions on Industrial Informatics, vol. 9, no. 3, pp. 1466-1476, Aug 2013.

[50] G. Bedi, G. K. Venayagamoorthy, R. Singh, R. R. Brooks, and K.-C. Wang, "Review of Internet of Things (IoT) in Electric Power and Energy Systems," IEEE Internet of Things Journal, vol. 5, no. 2, pp. 847-870, Apr 2018.

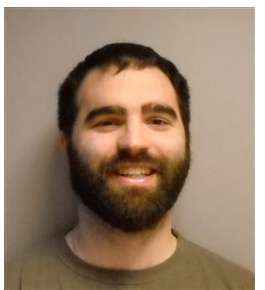

Markel Iglesias-Urkia is a $\mathrm{PhD}$ candidate at IK4Ikerlan on the Cybersecure-IoT team since 2016. He received his BSc on Computer Systems Engineering, BSc Computer Science \& Engineering and MSc on Embedded System Engineering from the University of the Basque Country. His research interests include Internet of Things (IoT), CoAP, lightweight protocols, Smart Grids, Cybersecurity and Web of Things. $\mathrm{He}$ is author or co-author of several peer-reviewed scientific publications in the field of Internet of Things.

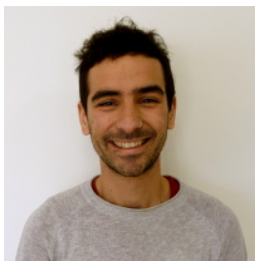

Dr. Diego Casado-Mansilla is Associate Researcher in the Societal Challenges research group from DeustoTech (University of Deusto). He received his $\mathrm{PhD}$ from the University of Deusto in 2016 in the topic of Persuasive Technologies for Sustainability and Behaviour change. His research interests are focused on Sustainable HCI, Persuasive and Behaviour change technologies, Sustainable design and Physical interaction with everyday objects. He has several relevant publications on the topics of sustainable HCI and Internet of Things (IoT).

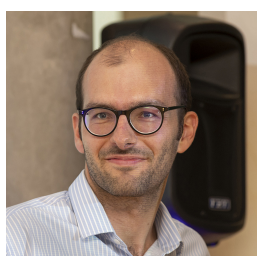

Prof. Dr. Simon Mayer is leading the chair for Interaction- and Communication-based Systems at the University of St. Gallen, CH. Before, he was a research group leader at the Austrian research center Pro ${ }^{2}$ Future, and a Senior Key Expert with the Siemens Web of Things research group in Berkeley, US. His research focuses on the integration of smart things into the Web, their semantic description, and supporting other machines as well as human users in finding and interacting with them. Simon graduated with a PhD in Computer Science from ETH Zurich.

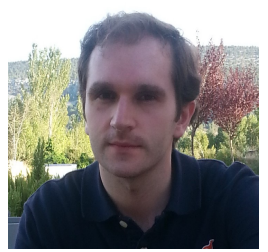

Dr. Josu Bilbao is with IK4-IKERLAN since 2003 where he is the team leader of the Cybersecure IoT team. He obtained the MsC in Telecommunication Engineering from the University of the Basque Country and the $\mathrm{PhD}$ degree in Computer Science from the University of Navarra. His current research interests are: reliable communications, realtime cyber-physical systems integration in IoT, Fogbased IoT architectures and 5G among others. He has taken part in different standardization bodies and platforms (IETF, IEEE, HANA, ETG, etc.)

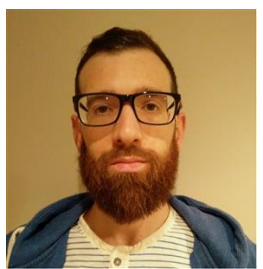

Dr. Aitor Urbieta is with IK4-IKERLAN since 2007 where he is a researcher at the CybersecureIoT team. He studied Informatics Engineering at the University of Mondragon, where he obtained his Ph.D. degree in Computer Science in 2010. His current research interests include Internet of Things, Fog Computing, Edge Computing, Smart Grids, Verification and Validation of IoT Environments.He is author or co-author several peer-reviewed scientific publications in the field of Internet of Things, Service Oriented Architectures and Semantic Web. 